\title{
M-RIP, a novel target of JNK signaling and a requirement for human cancer cell invasion
}

\author{
RYOKO ONO $^{1,2}$, JUNJI MATSUOKA ${ }^{1}$, TOMOKI YAMATSUJI ${ }^{1}$, YOSHIO NAOMOTO ${ }^{1}$, \\ NORIAKI TANAKA $^{1}$, HIDEKI MATSUI ${ }^{2}$ and MASAYUKI MATSUSHITA ${ }^{2,3}$ \\ Departments of ${ }^{1}$ Gastroenterological Surgery ans ${ }^{2}$ Physiology, Graduate School of Medicine and \\ Dentistry, Okayama University, 2-5-1 Shikata-cho, Okayama 700-8558; ${ }^{3}$ Mitsubishi Kagaku Institute \\ of Life Sciences, 11 Minamiooya, Machida, Tokyo 194-8511, Japan
}

Received March 11, 2008; Accepted May 16, 2008

DOI: 10.3892/ijmm_00000009

\begin{abstract}
Cell motility is involved in physiological and pathological processes such as the invasion and migration of cells. c-Jun N-terminal kinase (JNK) cascades are involved in the invasion and metastasis of cancer cells. However, little is known about the downstream signaling of JNK. In the present study, we used small interfering RNA (siRNA) directed against JNK1 to reduce its expression. We used microarray techniques to compare the gene expression profiles of epidermal growth factor (EGF)-stimulated HeLa cells with and without JNK1 siRNA treatment. We identified a JNK1 target gene, myosin phosphatase-Rho interacting protein (M-RIP). RNA interference-mediated inhibition of JNK1 strongly inhibited M-RIP mRNA expression induced by EGF, as well as the invasion of HeLa cells. In addition, M-RIP siRNA-treated cells showed significantly reduced invasive activity. Thus, a functional analysis of JNK1 and M-RIP with RNA interference reveals a critical role for this cascade in the invasive behavior of cancer cells.
\end{abstract}

\section{Introduction}

The migration and invasion of neoplastic cells is a complex process that involves microfilament and microtubule reorganization, membrane extension, adhesion and retraction $(1,2)$. One fundamental component of tumor cell invasion is increased cell migration. Epidermal growth factor (EGF)

Correspondence to: Dr Masayuki Matsushita, Department of Physiology, Okayama University Graduate School of Medicine and Dentistry, 2-5-1 Shikata-cho, Okayama 700-8558, Japan

E-mail: masayuki@mitils.jp

Abbreviations: FAK, focal adhesion kinase; JNK, Jun N-terminal kinase; M-RIP, myosin phosphatase-Rho interacting protein; eIF4E, eukaryotic initiation factor $4 \mathrm{E}$; RNAi, RNA interference; siRNA, small interfering RNA; qRT-PCR, quantitative reverse transcript polymerase chain reaction

Key words: focal adhesion kinase, Jun, myosin phosphatase-Rho interacting protein, eukaryotic initiation factor, small interfering RNA receptor activation is crucial for stimulating cell migration through receptor phosphorylation and the subsequent activation of downstream signaling pathways, including phospholipase $\mathrm{C}$ and the MAP kinase cascade $(3,4)$. However, additional signaling inputs through extracellular matrix-integrin interactions also play important roles in modulating EGF-stimulated cell motility responses (5). Focal adhesion kinase (FAK), a nonreceptor protein tyrosine kinase (PTK) which localizes to focal contact sites, is involved with both EGF receptors and integrin-associated signaling complexes (6). FAK has been linked to the generation of signals for cell survival, cell cycle progression, and cell motility $(3,6)$. FAK promotes both integrin-stimulated and growth factor-stimulated cell motility. Several signaling pathways which control cell migration, including the FAK pathway, converge at the c-Jun N-terminal kinases (JNKs) $(3,7)$. The JNK family plays fundamental roles in inflammation, differentiation and apoptosis. In addition, JNK pathways (especially JNK1) have recently been implicated in the regulation of cell migration via the phosphorylation of nuclear transcription factor c-Jun/AP-1, paxillin and microtubule-associated protein (MAP) (8-12).

Our goal in the present study was to identify additional downstream target genes for JNK1 in promoting EGFstimulated cell motility. To determine the genes that play a role in the JNK1 pathway, we used DNA microarray techniques to analyze the changes in gene expression in JNK1 siRNA-treated cells and control cells during EGF stimulation. We found that EGF stimulation significantly increased myosin phosphatase-Rho interacting protein (M-RIP) mRNA levels. This increase was specifically inhibited by JNK1 siRNA treatment. Both JNK1 and M-RIP siRNA treatment inhibited cell invasion through a Matrigel membrane. Our results indicate that EGF promotes cell invasion in part through the upregulation of M-RIP mRNA by the JNK1 cascade.

\section{Materials and methods}

Cell culture. HeLa cells were obtained from Dainippon Sumitomo Pharma (Tokyo, Japan) and cultured according to the manufacturer's instructions. DMEM medium was supplemented with $10 \% \mathrm{FBS}$, penicillin (100 units/ml) and streptomycin $(100 \mu \mathrm{g} / \mathrm{ml})$. 
Transfection procedures. Transfection was performed with Lipofectamine 2000 (Invitrogen, Life Technology) according to the manufacturer's instructions. After $6 \mathrm{~h}$ of incubation, the medium containing Lipofectamine was replaced with normal culture medium. Human FAK, JNK1 and M-RIP siRNA sense strands were designed as follows: FAK-1-siRNA, 5'-AGU GUGCUCUUGGUUCAAGdTdT-3'; FAK-2-siRNA, 5'-CGG ACAAGGGCUGUAAUCCdTdT-3'; JNK1-siRNA, 5'-GGA AUAGUAUGCGCAGCUUdTdT-3'; and M-RIP-siRNA, 5'-GUGUCAGAACUGCUUCAAGdTdT-3'.

Western blot analysis. Whole HeLa cell lysates were prepared by homogenization in lysis buffer [50 mM Tris-HCl, $\mathrm{pH} 7.4$, $1 \%$ Triton $\mathrm{X}-100,0.2 \%$ sodium deoxycholate and $0.2 \%$ sodium dodecyl sulfate (SDS)]. Tissue debris was removed by centrifugation, and the total protein concentration was determined using a BCA Protein Assay Reagent Kit (Pierce Chemical Company, Rockford, IL). Ten micrograms of protein from each sample was boiled for $5 \mathrm{~min}$ in $1 \mathrm{X}$ SDS sample buffer $(50 \mathrm{mM}$ Tris- $\mathrm{HCl}, \mathrm{pH} 6.8,12.5 \%$ glycerol, $1 \%$ SDS and $0.01 \%$ bromophenol blue) containing $5 \%$ ß-mercaptoethanol and separated by electrophoresis on SDSpolyacrylamide gels. Proteins were then transferred to nitrocellulose membranes (Hybond C, GE Healthcare UK Ltd., Little Chalfont, Buckinghamshire, UK), and immunological detection was performed with goat polyclonal antibodies to FAK, JNK1 and actin at a 1:1000-1:2000 dilution (Santa Cruz Biotechnology, Santa Cruz, CA) and anti-goat secondary antibodies conjugated to horseradish peroxidase at a dilution of 1:2000-1:4000 (Santa Cruz Biotechnology). Detection was performed with an Enhanced Chemiluminescence (ECL) Western Blotting Kit (GE Healthcare UK Ltd.).

Microarray analysis. Total RNA from HeLa cells was extracted by using an RNeasy Micro Kit (Qiagen, Cologne, Germany) following the manufacturer's instructions. Differential expression of genes in HeLa cells was examined by microarray techniques, according to the Affymetrix array protocol (Hokkaido Bioscience Inc., Hokkaido, Japan).

Quantitative PCR analysis. Quantitative PCR amplification and detection were performed by using SYBR Premix Ex Taq Mixes (Takara, Tokyo, Japan) and a Roche light cycler (Roche Diagnostics K.K., Tokyo, Japan) according to the manufacturers' instructions. The specific primer sequences were designed as follows: Actin-S, 5'-CCTCATGAAGATC CTCACCGA-3'; Actin-AS, 5'-TTGCCAATGGTGATGAC CTGG-3'; M-RIP-S, 5'-TTTATGGCGGTTGGCTGCTCC-3'; M-RIP-AS, 5'-CCTCATTTCTTCCTGCCAGAG-3'; eIF4E-S, 5'-GTTCCTGGCCCACTCTGTAA-3'; and eIF4E-AS, 5'-CC TGGATCCTTCACCAATGT-3'. Cycling parameters were $95^{\circ} \mathrm{C}$ for $15 \mathrm{~min}$, then 50 cycles of $95^{\circ} \mathrm{C}$ for $15 \mathrm{sec}$ and $60^{\circ} \mathrm{C}$ for $1 \mathrm{~min}$. As a control, the mRNA level of actin was determined in real-time PCR assays for each RNA sample and was used to correct for experimental variations. Melting curves were performed by using dissociation curves to ensure that only a single product was amplified. Quantifications were performed in duplicate, and the experiments were repeated independently 3 times. Expression levels were normalized to individual actin mRNA levels.
Cell migration assay. To perform a wound-healing assay, HeLa cells were plated onto collagen- or fibronectin-coated cover slips. The monolayer cells were then scratched manually with a plastic pipette tip. After being washed with phosphatebuffered saline, wounded monolayers of the cells were allowed to heal for 12 to $24 \mathrm{~h}$.

Cell invasion assay. Invasion was measured with an $8-\mu \mathrm{m}$ pore, 24-well Matrigel-coated Transwell chamber assay (Becton Dickinson Discovery Labware, Bedford, MA) according to the manufacturer's instructions. Twenty-four hours before the invasion assay was performed, HeLa cells were transfected with siRNA by Oligofectamine. These siRNAtreated HeLa cells $\left(2.5 \times 10^{4}\right)$ suspended in serum-free medium containing $0.1 \%$ bovine serum albumin were applied to the upper chamber and allowed to migrate to the lower side of the upper chamber for 2 to $12 \mathrm{~h}$. The cells that migrated to the lower side of the upper chamber were stained by Hoechst stain and counted. Cell migration was expressed as the number of migrated cells in one field (x10) of a fluorescence microscope. Five fields were randomly selected from all regions of a chamber. For all conditions, a minimum of 5 replicate chambers was assayed.

Data analysis. Differences between the control group and the obese group were analyzed with the Student's t-test performed with SPSS version 10.0 software. A two-sided P-value $<0.05$ was considered statistically significant. All values were expressed as the mean $\pm \mathrm{SD}$.

\section{Results}

Suppression of FAK by siRNA inhibited migration and invasion. We investigated cell migration and invasion after cells were treated with siRNA to inhibit the expression of FAK. To validate the assay system, HeLa cells were monitored in the presence of FAK siRNAs and a control siRNA (Fig. 1). FAK plays a central role in the motility and invasion of cancer cells, including HeLa cells. We first examined the knockdown efficiency of FAK by immunoblotting. Two types of FAKspecific siRNAs strongly inhibited protein expression levels compared with the control siRNA (Fig. 1A). By using scratch and invasion assays, FAK siRNA-treated HeLa cells were found to have significantly inhibited invasion and migration activity (Fig. 1B and C). The FAK siRNA did not affect cell viability (data not shown).

Suppression of JNK1 inhibited cell invasive activity. JNKs play crucial roles in cell migration and several developmental processes $(13,14)$. Stimulation of FAK with EGF activates the MEKK, MEK and JNK cascade and induces cell migration and invasion (3). To investigate the nature of FAK/JNK signaling, we designed selective JNK1 knockdown siRNA to discover the downstream targets of JNK1. JNK1 siRNA strongly and specifically inhibited JNK1 protein levels (Fig. 2A). We examined the invasive activity of cells treated with JNK1 siRNA, and we compared it to the invasiveness of cells treated with FAK siRNA and cells treated with a JNK inhibitor, SP600125. JNK1 inhibition with siRNA significantly reduced the invasive activity (Fig. 2B). 
A

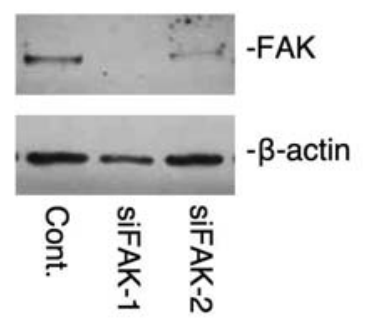

B
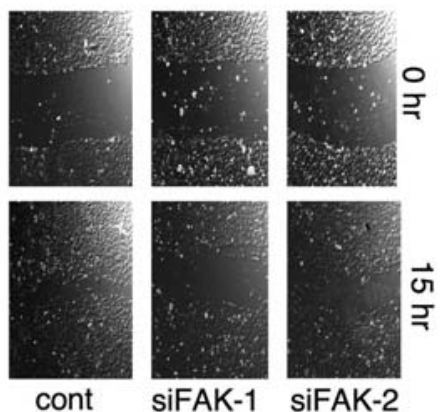

C
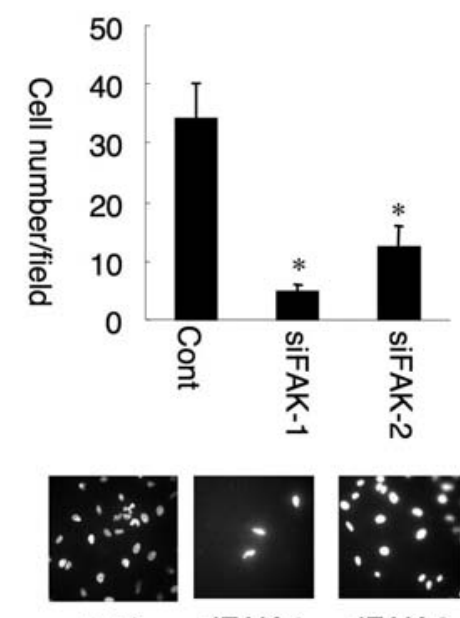

cont

siFAK-2

Figure 1. Decrease in FAK protein and cell motility after siRNA-mediated knockdown. (A) Western blots of HeLa cell lysates collected $48 \mathrm{~h}$ after transfection with control siRNA (Cont) and two types of FAK siRNA (siFAK-1, siFAK-2). Blots were probed with antibodies to FAK and actin. (B) Scratch migration assay of HeLa cells treated with siFAK-1 and siFAK-2. (C) Downregulation of FAK decreased HeLa cell invasion. Cells transiently transfected with siRNA were subjected to Boyden chamber assays of cell invasion through a Matrigel membrane. The data were collected from 5 individual fields of view (x10) of 5 replicate chambers. Representative images are shown below the graph. ${ }^{*} \mathrm{P}<0.01$.

Gene expression profile of JNK1 knockdown in EGF-treated HeLa cells. We used a microarray analysis to compare the gene expression profiles of HeLa cells with and without JNK1 siRNA treatment which were stimulated with EGF. We identified several genes specifically upregulated by EGF treatment and inhibited by JNK1 siRNA (Fig. 3A).

A

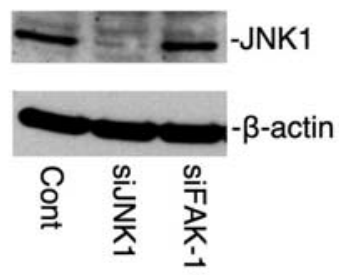

B

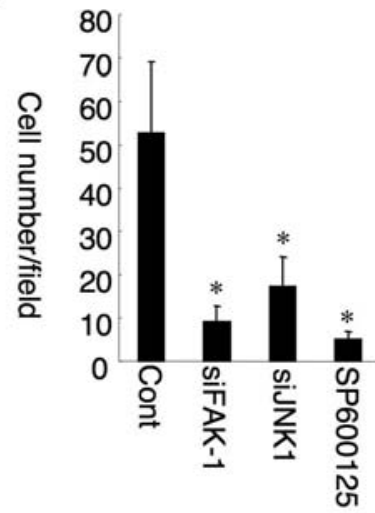

Figure 2. Decrease in JNK1 protein and cell invasion after siRNA-mediated knockdown. (A) Western blots of HeLa cell lysates collected $48 \mathrm{~h}$ after transfection with control siRNA (Cont), JNK1 siRNA (siJNK1) and FAK siRNA (siFAK-1). Blots were probed with antibodies to JNK1 and actin. (B) Downregulation of FAK-1, downregulation of JNK1, and a JNK inhibitor (SP6001259) decreased HeLa cell invasion. Cells transiently transfected with siRNA were subjected to Boyden chamber assays of cell invasion through a Matrigel membrane. Data were collected from 5 individual fields of view (x10) of 5 replicate chambers. Representative images are shown below the graph. ${ }^{*} \mathrm{P}<0.01$.

Validation of M-RIP and eIF4E by quantitative RT-PCR. Next, we determined the M-RIP mRNA levels during EGF stimulation by quantitative RT-PCR (qRT-PCR; Fig. 3). The M-RIP gene was upregulated by EGF treatment, and this upregulation was reduced to normal levels in JNK1 siRNAtreated HeLa cells (Fig. 3B). We also examined the eIF4E mRNA level by qRT-PCR and found that it was not reduced by JNK1 siRNA treatment (Fig. 3C).

Suppression of M-RIP inhibited HeLa cell invasive activity. Finally, we examined the role of M-RIP in cancer cell invasion. HeLa cells were transfected with control siRNA, JNK1 siRNA and M-RIP siRNA, and invasive activity was examined by a chamber assay. M-RIP siRNA reduced the M-RIP mRNA levels by $70 \%$ in comparison to control siRNA (Fig. 4A). M-RIP siRNA-treated cells displayed invasive activity which was significantly reduced in comparison with that of control siRNA-transfected cells (Fig. 4B).

\section{Discussion}

Initial studies of JNKs examined their ability to induce the phosphorylation of several transcription factors, such as the oncogene c-Jun. However, recent evidence indicates that JNKs have conserved their evolutionary role in cell movement, cytoskeleton rearrangement and migration. The ability of 
A

\begin{tabular}{|c|c|c|}
\hline Gene & $\frac{\text { EGF }}{\text { No treat }}$ & $\frac{\text { siJNK1+EGF }}{\text { EGF }}$ \\
\hline FABP5 & 1.6 & 0.32 \\
\hline M-RIP & 1.61 & 0.36 \\
\hline AQP1 & 1.5 & 0.47 \\
\hline eIF-4E & 1.62 & 0.73 \\
\hline
\end{tabular}

B

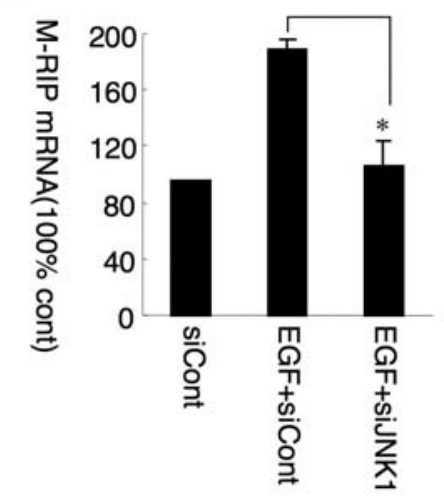

C

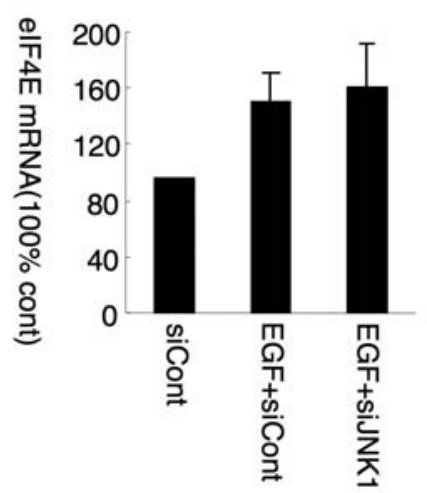

Figure 3. M-RIP mRNA level was regulated by EGF through the JNK1 pathway. (A) Microarray analysis of control (No treat) vs EGF stimulation, and EGF stimulation vs EGF stimulation plus siJNK1 treatment in HeLa cells. HeLa cells were incubated for $24 \mathrm{~h}$ in conditioned medium with siCont and siJNK1. These siRNA-treated cells were stimulated by incubation with EGF $(100 \mathrm{ng} / \mathrm{ml})$ for $8 \mathrm{~h}$, and then mRNA was collected for microarray analysis. FABP5, fatty acid binding protein 5; AQP1, aquaporin 1; eIF-4E, eukaryotic initiation factor 4E. (B) HeLa cells were incubated for $24 \mathrm{~h}$ in a conditioned medium with siControl (siCont) and siJNK1. These siRNAtreated cells were stimulated by incubation with EGF (100 ng/ml) for $8 \mathrm{~h}$. Then, mRNA was collected to analyze the mRNA levels by qRT-PCR. The M-RIP mRNA level was significantly upregulated by EGF ( $\left.{ }^{*} \mathrm{P}<0.05\right)$, and EGF stimulation did not increase the M-RIP mRNA level in JNK1 siRNAtreated HeLa cells. (C) The eIF4E mRNA level was significantly upregulated by EGF (0.05), and JNK 1 knockdown did not effect eIF4E mRNA regulation.

tumor cells to migrate from the primary tumor to surrounding tissues is a prerequisite for metastasis. JNK promotes increased motility of multiple cell types including tumor cells (8-12). In addition, the inhibition of JNK by siRNA, an inhibitor (SP60025) or gene deletion significantly impairs the rate of migration of several different cell types $(10,12-14)$.
A

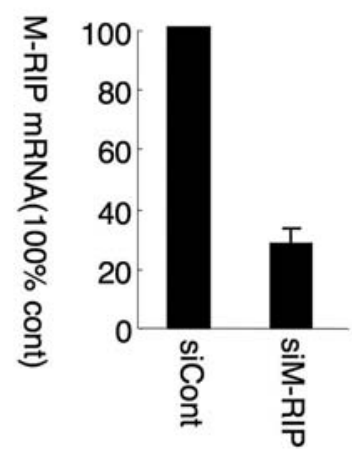

B

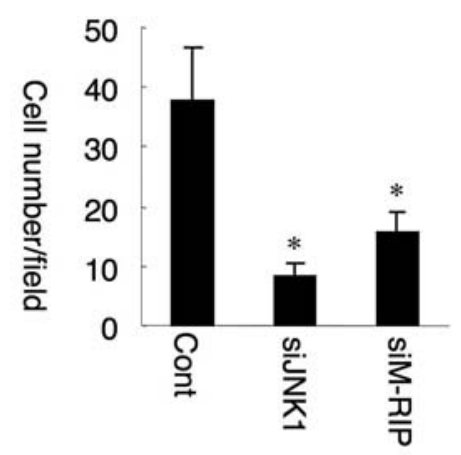

Figure 4. M-RIP inhibition strongly inhibited cell invasion. (A) Downregulation of M-RIP mRNA treated with siRNA for M-RIP (siM-RIP). Cells transiently transfected with siCont and siM-RIP were subjected to qRTPCR. (B) Downregulation of M-RIP and decreased HeLa cell invasion. Cells transiently transfected with siRNAs were subjected to Boyden chamber assays of cell invasion through a Matrigel membrane. The data were collected from 5 individual fields of view (x10) of 5 replicate chambers. Representative images are shown below the graph. ${ }^{*} \mathrm{P}<0.01$.

The signaling molecules involved in JNK pathways are also essential for cell migration. Upstream from JNK pathways, elements such as EGF receptors, FAK/Src, MEK kinase 1 and MKK4/7 are essential for cell migration and development (3). Additionally, JNK substrates such as paxillin, spir and MAP2 are directly involved in cell motility. However, the transcriptional regulation of cell invasion through the phosphorylation of transcription factors such as Jun by JNK has not been clearly studied. To address the underlying molecular mechanisms involved in the JNK1 pathway implicated in cancer cell motility, we used a microarray analysis to compare the expression profiles of EGF-stimulated HeLa cells with and without JNK1 siRNA treatment.

The inhibition of the JNK1 function by siRNA resulted in a decreased level of M-RIP transcription induced by EGF, as well as inhibition of HeLa cell invasion through a reconstituted basement membrane (Figs. 3 and 4). M-RIP was initially identified as a $116-\mathrm{kDa}$ human protein that interacts with both myosin phosphatase and RhoA $(15,16)$. p116M-RIP colocalizes with both stress fibers and lamellapodia, has actin-bundling properties in vitro, and causes actin filament disassembly when overexpressed $(17,18)$. In neurons, M-RIP is essential for neurite outgrowth and may act as a scaffold to target the myosin phosphatase complex to the actin cyto- 
skeleton (15). These data strongly suggest that M-RIP regulates cell motility through actin regulation. Our data suggest that M-RIP transcription is regulated by the JNK1 pathway. We therefore conclude that EGF can potentially affect M-RIP transcription through the JNK1 regulatory pathway. We propose that JNK1 activation in vivo may upregulate M-RIP transcription, facilitating an invasive phenotype.

\section{Acknowledgements}

We thank T. Ogawa for his technical assistance. This work was supported, in part, by grants-in-aid for Scientific Research from the Ministry of Education, Culture, Sports, Science and Technology of Japan.

\section{References}

1. Friedl $\mathrm{P}$ and Wolf $\mathrm{K}$ : Tumour-cell invasion and migration: diversity and escape mechanisms. Nat Rev Cancer 3: 362-374, 2003.

2. Carragher NO and Frame MC: Focal adhesion and actin dynamics: a place where kinases and proteases meet to promote invasion. Trends Cell Biol 14: 241-249, 2004.

3. Huang C, Jacobson K and Schaller MD: MAP kinases and cell migration. J Cell Sci 117: 4619-4628, 2004.

4. Bill HM, Kundsen B, Moores Sl, et al: Epidermal growth factor receptor-dependent regulation of integrin-mediated signaling and cell cycle entry in epithelial cells. Mol Cell Biol 24: 8586-8599, 2004.

5. Sieg DJ, Hauck CR, Ilic D, et al: FAK integrates growth-factor and integrin signals to promote cell migration. Nat Cell Biol 2: 249-256, 2000.

6. Mitra Sk, Hanson DA and Schaepfer DD: Focal adhesion kinase: in command and control of cell motility. Nat Rev Mol Cell Biol 6: 56-68, 2005.

7. Xia Y and Karin M: The control of cell motility and epithelial morphogenesis by Jun kinases. Trends Cell Biol 14: 94-101, 2004 .
8. Clarke N, Arenzana N, Hai T, et al: Epidermal growth factor induction of the c-jun promoter by a Rac pathway. Mol Cell Biol 18: 1065-1073, 1998.

9. Westermarck J, Li S, Jaakkola P, et al: Activation of fibroblast collagenase- 1 expression by tumor cells of squamous cell carcinomas is mediated by p38 mitogen-activated protein kinase and c-Jun NH2-terminal kinase-2. Cancer Res 60: 7156-7162, 2000 .

10. Huang C, Rajfur Z, Borchers C, et al: JNK phosphorylates paxillin and regulates cell migration. Nature 424: 219-223, 2003.

11. Malliri A, Symons M, Hennigan RF, et al: The transcription factor AP-1 is required for EGF-induced activation of rho-like GTPases, cytoskeletal rearrangements, motility, and in vitro invasion of A431 cells. J Cell Biol 143: 1087-1099, 1998.

12. Collins CS, Hong J, Sapinoso L, et al: A small interfering RNA screen for modulators of tumor cell motility identifies MAP4K4 as a promigratory kinase. Proc Natl Acad Sci USA 103: 3775-3780, 2006 .

13. Li G, Gustafson-Brown C, Hanks SK, et al: c-Jun is essential for organization of the epidermal leading edge. Dev Cell 4: 865-877, 2003.

14. Zenz R, Scheuch H, Martin P, et al: c-Jun regulates eyelid closure and skin tumor development through EGFR signaling. Dev Cell 4: 879-889, 2003.

15. Gebbink MF, Kranenburg O, Poland M, et al: Identification of a novel, putative Rho-specific GDP/GTP exchange factor and a RhoA-binding protein: control of neuronal morphology. J Cell Biol 137: 1603-1613, 1997.

16. Surks HK, Riddick N and Ohtani K: M-RIP targets myosin phosphatase to stress fibers to regulate myosin light chain phosphorylation in vascular smooth muscle cells. J Biol Chem 280: 42543-42551, 2005.

17. Mulder J, Ariaens A, van den Boomen, et al: p116Rip targets myosin phosphatase to the actin cytoskeleton and is essential for RhoA/ROCK-regulated neuritogenesis. Mol Biol Cell 15: 5516-5527, 2004

18. Surks HK, Richards CT and Mendelsohn ME: Myosin phosphatase-Rho interacting protein. A new member of the myosin phosphatase complex that directly binds RhoA. J Biol Chem 278: 51484-51493, 2003. 